\title{
Tratamiento temprano de alteraciones orofaciales con fisioterapia y placa palatina en niños con síndrome de Down
}

\author{
Early treatment of orofacial alterations with physical therapy \\ and palatal plate in children with Down syndrome
}

Paula Vivar Vergara' ${ }^{1}$ ORCID: 0000-0002-9411-3019

Fernanda Riveros Figueroa ${ }^{2}$ ORCID: 0000-0001-8842-2205

Germán Sepúlveda Hidalgo² ORCID:0000-0001-8028-9796

María Antonieta Pérez Flores ${ }^{3}$ ORCID: 0000-0002-2481-7088

Claudia Fierro Monti' ORCID: 0000-0002-0308-6044

\section{Resumen}

El Síndrome de Down (SD) o trisomía 21, es la anomalía congénita autosómica más frecuente cuya prevalencia mundial es de 10/10.000, en Chile de 2,5/1.000 nacidos vivos. Por trastornos de crecimiento, desarrollo e hipotonía muscular, las personas con SD presentan boca abierta con gran salivación, labio inferior evertido, elevación del labio superior en inactividad y descenso del ángulo de la boca.

Objetivo: Evaluar la literatura sobre el tratamiento temprano de alteraciones orofaciales en niños con SD para prevenirlas o minimizarlas.

Método: Búsqueda en base de datos PubMed y Scielo, sin discriminar año de publicación, idioma: inglés-español, seleccionando 26 artículos.

Resultados: Existen cambios positivos en la función motora orofacial, observando mayores cambios en los casos más severos.

Conclusiones: Un niño con SD se beneficia del tratamiento temprano, pero faltan estudios comparables en cuanto a duración, edad y tipo de terapia.

Palabras clave: síndrome de down, maloclusión, modalidades de terapia física.

Facultad de Odontología, Universidad de Valparaíso, Universidad de Concepción, Chile.

Facultad de Odontología. Universidad de Concepción, Chile.

Departamento de Pediatría Bucal, Facultad de Odontología, Universidad de Concepción, Chile.

Fecha de recibido: 14.04.2019 - Fecha de aceptado: 26.07.2019 


\section{Abstract}

Down syndrome (DS) or trisomy 21 is the most frequent autosomal congenital anomaly, with a worldwide prevalence of 10/10,000; in Chile, the prevalence is $2.5 / 1,000$ live births. People with DS present an open mouth with increased salivation, an everted lower lip, elevated and inactive upper lip, and lowering of the angle of the mouth on account of growth and development disorders, and muscular hypotonia. Objective: To evaluate the literature on the early treatment of orofacial alterations in children with DS to prevent or minimize them. Method: Search in PubMed and Scielo databases, regardless of the year of publication or language; 26 articles were selected. Results: There are positive effects on the orofacial motor function, observing significant changes in the most severe cases. Conclusions: A child with DS can benefit from early treatment, but there are insufficient comparable studies in terms of duration, age and type of therapy.

Keywords: down syndrome, malocclusion, physical therapy modalities.

\section{Introducción}

El síndrome de Down (SD), denominado también trisomía 21, trisomía G o mongolismo, representa la anomalía congénita autosómica más frecuente. Aproximadamente el $95 \%$ de los casos con SD se debe a la adición de un cromosoma 21, es decir, presentan una dotación de 47 cromosomas en vez de 46 . El otro 5\% es causado por otras anomalías cromosómicas, siendo un $3 \%$ por translocación y un $2 \%$ por mosaicismo o trisomía parcial.

La primera descripción de un niño que supuestamente tenía trisomía 21, fue

\section{Resumo}

A síndrome de Down (SD) ou a trissomia do cromossomo 21 , é a anomalia congênita autossômica mais frequente, com prevalência global de $1 / 1.000$, no Chile de $2.5 / 1.000$ nascidos vivos. Devido aos distúrbios de crescimento, desenvolvimento e hipotonia muscular, as pessoas com SD apresentam boca aberta com grande salivação, lábio inferior evertido, elevação do lábio superior inativo e ângulo da boca mais baixo. Objetivo: Avaliar a literatura sobre o tratamento precoce dos distúrbios orofaciais em crianças com SD para prevenilas ou minimizá-las. Método: Pesquisa na base de dados PubMed e Scielo, sem discriminar ano de publicação, idioma: inglês-espanhol, selecionando 26 artigos. Resultados: Existem mudanças positivas na função motora orofacial, observando-se grandes alteraçóes nos casos mais graves. Conclusóes: Uma criança com $\mathrm{SD}$ se beneficia do tratamento precoce, mas faltam estudos comparáveis em termos de duração, idade e tipo de terapia.

Palavras-chave: síndrome de down, má oclusão, modalidades fisioterapêuticas. 
A nivel mundial, la prevalencia global de SD es de 10 por cada 10.000 nacidos vivos. Pero hay importantes diferencias entre países, y éstas dependen principalmente de sus variables socioculturales. En países donde el aborto es ilegal, como ocurre en Irlanda o en los Emiratos Árabes Unidos, la prevalencia es mayor: entre 17 y 31 por 10.000 nacimientos vivos; en Estados Unidos 1 de cada 732 bebés, mientras que la tasa de prevalencia es de 1.08 por cada 1.000 nacidos vivos en el Reino Unido. En Francia, en cambio, la prevalencia baja a 7.5 por 10.000 , debido quizás a la alta tasa de aborto $(77 \%)^{(3)}$. En Latinoamérica se ha observado un aumento de las tasas de prevalencia de SD desde 1974 hasta el 2005. El International Clearinghouse for Birth Defects Monitoring Systems obtuvo una tasa de 1,46 por 1000 nacimientos en el periodo 1974-1979, alcanzando un 1,85 en 1998. Además, el Estudio Colaborativo Latinoamericano de Malformaciones Congénitas (ECLAMC) dio para el periodo 1998-2005 una tasa global de 1,88 . Bajo este promedio se encuentra Brasil 1,72, Colombia 1,72, Bolivia 1,55, Venezuela 1,49, Ecuador 1,48 y Uruguay 1.32. Y sobre este promedio están las tasas de Argentina 2.01, Paraguay 1.98 y Chile quien presenta la tasa más alta de Sudamérica con un 2.47 por 1.000 nacidos vivos ${ }^{(3)}$.

La prevalencia no tiene predilección racial, socioeconómica ni de género, pero el aumento de la edad materna se asocia con una mayor probabilidad de dar a luz un hijo con trisomía 21. A los 35 años ya se observa un aumento del riesgo, siendo considerablemente mayor después de los 45 ańos, alcanzando una tasa de 1 en 25 nacidos vivos ${ }^{(4)}$.

El SD presenta variadas características, alteraciones y/o manifestaciones clínicas, tanto sistémicas como craneofaciales, expresadas individualmente en distintos grados. Dentro de las anomalías sistémicas se encuentran las cardiovasculares, hematopoyéticas, nerviosas, musculoesqueléticas, entre otras. Es por ello que desde el nacimiento todos los niños con SD deben ser sometidos a múltiples exámenes y evaluaciones que puedan detectar a tiempo ciertas alteraciones, y durante todo su crecimiento deben estar en constantes visitas médicas y odontológi$\operatorname{cas}^{(5,6)}$.

Las personas con SD expresan un fenotipo distintivo, que se debe en parte al resultado de los trastornos de crecimiento, desarrollo óseo e hipotonía muscular generalizada que presentan estos individuos ${ }^{(6,7)}$. Padeciendo múltiples trastornos orofaciales genéticamente determinados, así como diversos grados de disfunciones del sistema estomatognático ${ }^{(4)}$. Algunas de estas características son facies de cráneo pequeño, depresión de la parte media de la cara y del hueso nasal, procesos malares planos y ojos inclinados hacia arriba. Con base craneal corta y plana, reducción de la longitud maxilar o hipoplasia maxilar y retrusión de la parte media de la cara. En general se describe un patrón esquelético clase III de Angle ${ }^{(8,9)}$, sin embargo, muchos aspectos de la morfología craneofacial siguen sin estar claros, como por ejemplo la anatomía detallada de la mandíbula (cuerpo, rama, y mentón) y las dimensiones alveolares. Así tenemos que algunos autores han descrito la mandíbula como pequeña, mientras que otros han encontrado que es similar a la de la población no afectada por el síndrome $e^{(10-12)}$.

Castillo Morales hace una diferenciación de los trastornos patológicos que padecen los pacientes con SD entre primarios y secundarios. Los trastornos primarios implican la hipotonía de los músculos orofaciales, reducción del tono del aparato ligamentoso de la articulación temporomandibular (ATM), lengua hipotónica protruida con diástasis en la línea media y frenillo lingual débil. Tercio medio facial disminuido por hipoplasia del maxilar, altura del paladar reducida, velo hipotónico, dentición retardada, microdoncia y agenesias. Las patologías secundarias ocurren por el mal funcionamiento de las estructuras orales y respiratorias, e incluyen una subluxación de la mandíbula, respiración bucal, infecciones de las vías respiratorias, ma- 
loclusiones y problemas de fonación. Dentro de los trastornos secundarios está también una expresión característica de boca abierta con gran salivación, el labio inferior evertido, elevación del labio superior inactivo y descenso del ángulo de la boca. Además la protrusión constante de la lengua no permite un correcto cierre bucal y genera una superficie lingual seca y agrietada, y los incisivos superiores e inferiores se protruyen ocasionando a veces una mordida abierta dentoalveolar ${ }^{(13-15)}$.

La terapia de regulación orofacial de CastilloMorales (TRO) desarrollada en 1978 en $\mathrm{Mu}-$ nich, Alemania, busca mejorar varias de las patologías primarias y secundarias comunes a los nińos con SD. La terapia consta de dos componentes, el primero es la estimulación física manual de la musculatura orofacial proporcionada generalmente por un fisioterapeuta, $y$ el segundo es la inserción de una placa palatina estimulante, removible, según sea la necesidad del caso. La placa palatina está diseñada para alterar la posición de reposo de la lengua, provocar movimientos específicos de ésta, aumentar la movilidad del labio superior y aumentar el tono de la musculatura facial ${ }^{(16)}$, lo que lleva a mejoras en el cierre bucal, succión, fonación, deglución y respiración nasal ${ }^{(4-15)}$.

Por lo descrito son muchos los factores que favorecen una maloclusión en niños con trisomía 21, por ello desde pequeños y en especial durante toda la etapa de crecimiento y desarrollo, deberían recibir atención odontológica multidisciplinaria que permita evaluar la secuencia de anomalías esperables que se puedan prevenir o intervenir en cada caso. El tratamiento ortopédico y ortodóncico desde los primeros meses de vida hasta la edad adulta puede corregir y controlar anomalías funcionales, así como el desarrollo de estructuras dentales y faciales ${ }^{(4-17)}$. Esta revisión tiene por objetivo evaluar la literatura sobre el tratamiento temprano con fisioterapia y placa palatina en niños con SD, con el fin de prevenir o minimizar las alteraciones orofaciales.

\section{Metodología}

Se ha realizado una revisión de la literatura científica basada en una metodología de búsqueda estructurada sobre las bases de datos PubMed y Scielo; Los términos utilizados para la búsqueda fueron "Maloclussion down syndrome", "Orthodontic treatment down syndrome", "Early orthodontic down syndrome", "Stimulating plate down syndrome", "prevention malocclusion down syndrome"; los cuales fueron combinados entregando como resultado de la búsqueda la cantidad de 180 artículos. Se incluyeron artículos en inglés y español, sin discriminar por el año de publicación. Además, se excluyeron los artículos repetidos, los que se referían a tratamientos realizados en adultos y los que tuvieran poca relevancia para el tema tratado.

De este modo, fueron considerados 26 artículos, de los cuales 10 hacen referencia a evaluación de tratamientos tempranos en niños con SD con el fin de prevenir o minimizar las alteraciones orofaciales, en especial, la terapia de regulación orofacial con placa palatina. Posteriormente se llevó a cabo una lectura crítica de la literatura encontrada, la síntesis y análisis de la información entregada por los artículos.

\section{Desarrollo}

La terapia de regulación orofacial de Castillo Morales (TRO) es la base de estudios que buscan evaluar alternativas de tratamiento temprano en niños con SD, con el fin de evitar y/o disminuir las alteraciones orofaciales, como la posición lingual y labial anormal. Las investigaciones posteriores desarrollaron y evaluaron tratamientos inspirados en la TRO, pero con modificaciones, teniendo en común la aplicación obligatoria de la fisioterapia y como variante principal el tipo de placa utilizada (según estuviera indicada), resultando más correcto llamarlo tratamiento temprano con 
fisioterapia (TTF) o tratamiento temprano con fisioterapia más placa palatina (TTFP).

La indicación del uso de una placa palatina estimulante se centra en casos de pacientes con: a) una lengua hipotónica amplia y en una posición interdental o interlabial durante varias horas al día; b) una lengua con diástasis y protrusión adicional; c) un labio superior hipotónico inactivo con lados estrechos ${ }^{(15-16-18)}$.

Ya en el año 1991, mediante un estudio en el que participaron 67 niños con trisomía 21, se evaluaron los resultados del TTFP, observando cambios positivos en la posición lingual y labial, corrigiéndose además la función motora en acciones tales como la succión y contención de la saliva; todo esto con un ańo de tratamiento, usando la placa 1 hora al día al principio, para luego avanzar a 3 o 4 horas diarias ${ }^{(15)}$. Usando el mismo régimen de tratamiento, pero con solo 10 meses de duración, se observaron mejorías en el cierre bucal, evidenciándose durante el examen clínico y encuesta realizada a los padres, que la boca permanece abierta durante menos tiempo en comparación al inicio de la terapia, sumado a notorias mejorías en la postura de la lengua, la cual ya no se encontraría fuera de la boca o entre los dientes la mayor parte del tiempo ${ }^{(18)}$.

Un estudio del año 2000 intentó incrementar la evidencia, contando con un grupo control, lo cual permitió evaluar y analizar los resultados de una forma diferente a lo que la literatura mostraba hasta la época, reconociendo esta carencia como una debilidad justificada en temas éticos ${ }^{(15)}$, dejando ver luego de 2 años de tratamiento, mejoras considerables en el cierre labial en el $65 \%$ de los individuos en estudio, versus un $8 \%$ del grupo control que sólo recibió terapia física. Algo similar ocurre con la posición lingual, la cual mejoró en el $55 \%$ de los niños del grupo de estudio frente al 30\% del grupo control ${ }^{(19)}$. Resultados similares fueron los publicados por Carlstedt, en un estudio del mismo tipo, pero con un tratamiento que se prolongó por 4 años, obteniendo efectos positivos sobre la función motora oral, como una mejora significativa en las siguientes variables: la actividad del labio, la posición de la lengua y la expresión facial ${ }^{(20-21)}$, resultados que muestran gran similitud a tratamientos más cortos, como los estudiados por el mismo autor, transcurridos 9 y 12 meses desde el examen inicial ${ }^{(22)}$. Reafirmando estos resultados, en el año 2004 un estudio que sigue el mismo protocolo de investigaciones anteriores (fisioterapia más placa palatina), indica que luego de 1 año de tratamiento se observaron cambios en la postura bucal en el $75 \%$ de un total de 20 niños en estudio, también un 65\% presento mejoras en la postura de la lengua ${ }^{(23)}$.

En los estudios ya mencionados, es recurrente la evaluación de los cambios en la postura labial, cierre bucal y posición de la lengua, sin embargo, hay investigaciones que evalúan además otros aspectos relevantes, como la persistencia de hábitos, erupción dentaria, oclusión y desarrollo del habla ${ }^{(20-21-24)}$. En relación a la erupción dentaria, Bäckman, et al. mostraron que los pacientes tratados sin placa palatina presentaron menos dientes erupcionados que los niños tratados con este aparato y que los niños sin SD. Posiblemente esto podría deberse a la estimulación de la mucosa oral por las placas palatinas acelerando la erupción dental ${ }^{24}$. También se ha indicado que el TTFP, desde los 6 a los 48 meses de edad (modificando la placa dependiendo del desarrollo del niño), puede prevenir alteraciones en la oclusión dentaria, observándose que la mordida cruzada posterior es significativamente menor en sujetos sometidos a tratamiento temprano, que en individuos pertenecientes al grupo control ${ }^{(24)}$. De igual forma un estudio observó cambios en el habla de niños sometidos a este tratamiento, donde las capacidades de este tipo son 3 veces mejores que en niños que no han recibido esta terapia ${ }^{(24)}$, en contraste con los nulos resultados favorables en este ítem mostrado en estudios del mismo tipo ${ }^{(20-21)}$.

En Chile, un estudio del año 2010 realizado por Padró et al., luego de aplicar el tratamiento con placa por 2 meses -tiempo muy acotado en rela- 
ción a lo sugerido por algunos autores ${ }^{(22)}$ observaron, a través de monitoreos semanales, cambios favorables menores y leves en la tonicidad de la lengua, cierre bucal, retracción lingual y postura labial, en una muestra de 5 niños con SD de 15 meses de edad en promedio ${ }^{(25)}$.

La edad en que es recomendable iniciar el tratamiento es también materia a considerar, puesto que las experiencias son muy variadas al respecto. Tratamientos iniciados a solo 1, 2 o 3 meses de edad han mostrado tener buenos resultados en la postura lingual, contacto labial y expresiones miméticas ${ }^{(4-20-23)}$, aun cuando puede parecer compleja la cooperación de un infante de esa edad. En el otro extremo, existen casos en los que el tratamiento ha sido iniciado incluso a los 5 años de edad ${ }^{(22-23)}$. Es importante considerar que existen estudios en los que la edad de los individuos participantes era bastante dispar, teniendo grandes diferencias entre sí, llegando a los 30 meses $^{(21)}$ o 59 meses de diferencia ${ }^{(23)}$. Un análisis de varianza determinó que no se muestran efectos relacionados con la edad en los resultados ${ }^{(15)}$.

En relación a la erupción dentaria según Castillo Morales, representa una dificultad en la ejecución de la terapia, debido a que se complican los métodos de retención del aparato, por lo que suspenderla durante ese periodo hasta la erupción total de las piezas dentarias, parece ser una alternativa con buenos resultados cuando el inicio del tratamiento es previo a esta etapa del desarrollo ${ }^{(18)}$.

Más escasos son los estudios a largo plazo que permitan evaluar y valorar la estabilidad de los resultados, después de varios años de terminada la terapia. Se ha observado que los efectos positivos encontrados al término del tratamiento mejoraron en lo que respecta a la postura habitual de la boca, aumentando el tiempo en que se mantuvo cerrada, y a la postura habitual de la lengua, aumentando el tiempo en que ésta se mantuvo dentro de la boca ${ }^{(18)}$. Korbmacher determinó que, luego de 12 años de finalizado el tratamiento, la postura de la boca mejoró en
$30 \%$ de los niños, se mantuvo en un $10 \%$ y se deterioró en un $45 \%$. No obstante los cambios desde el inicio del tratamiento se describen con un $55 \%$ de mejorías en este ítem y un $40 \%$ sin cambios. En el aspecto de postura de la lengua, los cambios desde el término de la terapia hasta el control posterior a 12 ańos, muestran un efecto positivo en el $20 \%$ de los niños, mantención en un $60 \%$ de los casos y un $5 \%$ de deterioro. Positivamente, los cambios desde el inicio del tratamiento hasta el examen transcurridos 12 años se dieron en el 75\% de los individuos y se mantuvo la condición en un $25 \%$, no existiendo efectos negativos ${ }^{(23)}$.

\section{Discusión}

Debido a la gran variabilidad de los elementos que componen el TTFP, es imposible atribuir efectos específicos a los diferentes componentes que constituyen este tratamiento, sin embargo, la inserción aislada de una placa estimulante sin terapia física adicional no está indicada. De hecho, en ninguno de los artículos revisados, en que se realizó tratamiento temprano, se utilizó de forma exclusiva la placa palatina. Los ejercicios manuales contribuyen al fortalecimiento de los músculos orofaciales y apoyan el efecto de estimulación mecánica de la placa, sin embargo, ningún estudio describe con precisión en qué consiste este entrenamiento físico ${ }^{(23)}$.

La severidad de los signos y síntomas de nińos con SD puede diferir enormemente entre uno y otro, es por eso que la inserción de una placa palatina estimulante es requerida sólo en un cuarto o un tercio de todos los pacientes. Los niños con disfunción orofacial leve, muestran un desarrollo más armonioso de la región orofacial per se, que los niños con disfunción orofacial más severa ${ }^{(23)}$, lo cual se refleja en los resultados obtenidos de los artículos revisados. El objetivo de la terapia con placa palatina es generar un cambio posicional de la lengua hacia arriba y atrás, combinado con el entrenamiento automático de los músculos y la estimulación del 
labio superior inactivo. La evidencia científica revisada ${ }^{(4-15-18-19-20-21-22-23-24-25)}$ concuerda en que la inserción de la placa palatina realmente produce una reacción que implica la retracción de la lengua a la parte posterior de la boca, justificando fuertemente el uso del TTFP. Sin embargo, para evaluar reales cambios en cuanto a la tonicidad muscular se requieren métodos objetivos que permitan cuantificarla.

Un punto crítico en cuanto a la metodología de los estudios es la gran cantidad de tipos de placas palatinas, basadas en su mayoría en el diseño de Castillo-Morales, a la cual se le aplican diversas modificaciones. Durante la emergencia de los dientes en boca, hubo algunos casos en que la placa se modificó utilizando sistemas de retención accesorios y otros en que simplemente se suspendió el tratamiento por algunos meses hasta una condición oral estable. El uso de diferentes diseños, no sólo varió entre los participantes de diferentes estudios, sino que dentro del mismo estudio e incluso en el mismo paciente, lo que conduce a mayor sesgo y no es posible determinar cuál es la mejor alternativa, sumando complejidad al realizar una comparación estandarizada de la técnica ${ }^{(22)}$.

Respecto a la estabilidad del tratamiento, existen pocos estudios con evaluaciones a largo plazo. Se ha estimado que un período de tratamiento de 1 año es demasiado corto para establecer conclusiones definitivas, sobre si el tratamiento tiene efectos duraderos en la función orofacial $^{(22-23)}$. Korbmacher $\mathrm{H}$, et al. efectúa el seguimiento de los pacientes por más de 12 años, lo cual ofrece un potencial predictivo mayor sobre la estabilidad a largo plazo de los efectos logrados durante el tratamiento. Este estudio concluyó que el desarrollo orofacial a largo plazo, depende de la gravedad de las características orofaciales en la primera infancia, antes de la inserción de la placa. Los niños con postura habitual de boca abierta -mayor a $10 \mathrm{~mm}$ - y posición lingual extraoral (características orofaciales severas) exhibieron un mejor desarrollo durante el período de seguimiento, que aquellos que presentaban habitualmente, un espacio interlabial menor a $10 \mathrm{~mm}$ y una posición interdental de la lengua (características orofaciales moderadas) al comienzo del TTFP $^{(23)}$. Esto demuestra que los cambios logrados en la apariencia orofacial se mantienen estables en la mayoría de los casos, a pesar que el estímulo mecánico de la placa palatina no esté presente durante el período de seguimiento $^{(23)}$. Esto coincide con Cudzilo et al., informando que el aumento de la tensión muscular también continuó después de que la placa se había eliminado ${ }^{(4)}$.

Si bien aún hay poca evidencia científica sobre qué tan estables son en el tiempo, los resultados que se obtienen de la combinación de la fisioterapia y la placa palatina, sí se puede reconocer que los beneficios que se observan directamente en la función muscular de la lengua y los labios, repercuten también en un mejor desarrollo del niño, observado por ejemplo, en funciones como: deglución, oclusión, erupción dentaria, masticación, habla y lenguaje (20-23-24). $^{\text {. }}$ El no tratamiento y la nula existencia de mejoras en las fuerzas musculares orofaciales anómalas perpetúan o exacerban un patrón esquelético clase III o una biprotrusión alveolar incisiva $^{(23)}$, ya que, las maloclusiones en niños con trisomía 21 aumentan o se agravan con la edad por el retraso del crecimiento craneofacial y la disfunción motora oral característica ${ }^{(20)}$. Sin embargo, las consecuencias del patrón de crecimiento hacia una clase III esqueletal con un resalte anterior negativo no logran evitarse con el TTFP ${ }^{(24)}$.

Varios estudios coinciden, que los resultados no son atribuibles a la terapia y que el efecto real de la función muscular sólo se puede evaluar a largo plazo. En investigaciones a corto plazo, se observan cambios en la posición de ciertos músculos, mientras que a largo plazo, se puede evaluar el efecto en el tono muscular y la estabilidad en el tiempo de los cambios, y así también, descartar que se haya tratado de una mejoría transitoria por la reciente aplicación de un elemento mecánico ${ }^{(21-25)}$. Después de 4 
años de terapia, Carlstedt et al., concluyen que la contracción labial durante el habla, puede implicar que la placa palatina ha obtenido un aumento del tono muscular en los $\operatorname{labios}^{(20)}$. Con respecto a los resultados del cuestionario aplicado a los padres, éstos muestran diferencias entre los grupos de pacientes tratados y no tratados en relación a hábitos de boca abierta, hábitos del sueño, ronquidos y apneas del sueño, habiéndose detectado menos ronquidos en el grupo de pacientes tratados con placa palatina. Es posible que esto dependa de una posición lingual mejorada durante el sueño, sin embargo, para poder comprobar que la placa realmente tiene efectos positivos sobre patrones de respiración durante el sueño, es necesario realizar investigaciones profundas y a largo plazo ${ }^{(20)}$.

Es cuestionable que ninguno de los métodos de evaluación utilizados en los estudios revisados esté estandarizado (examen clínico, grabación/ registro de video, fotografías y cuestionario para padres). El resultado del TTFP es difícil de medir objetivamente ${ }^{(19)}$, y la evaluación visual sigue siendo uno de los métodos más importantes para medir el progreso del tratamiento temprano, es decir, para registrar los cambios en la tensión muscular mimética, posición de la lengua y cierre del labio, durante y después del tratamiento ${ }^{(19)}$. Haberfellner y Richter (1980), Stratton (1981), coinciden que el método aceptado para documentar los efectos del tratamiento sigue siendo la observación clínica ${ }^{(15)}$. Se considera que una grabación de video de la cara del niño es un método adecuado para evaluar la función muscular orofacial, dando la posibilidad de repetir el análisis, aunque la grabación refleja la función oral durante un período muy limitado y por lo tanto puede ser influenciada por la fatiga, el estado de ánimo del niño o condiciones ambientales como la posición de la cámara y rayo, hora del día, etc. ${ }^{(19-22)}$. Otros estudios incluyen como complemento la aplicación de un cuestionario a los padres en cada sesión de control, considerando que este procedimiento puede verse influenciado por las expectativas paternas con un resultado inapropiadamente positivo $^{(4-16-18-19)}$. Por otro lado, la evaluación de fotografías frontales de bebés y niños pequeños es muy difícil, debido a que no es posible obtener una posición estandarizada ${ }^{(19)}$. El examen clínico y el registro de video parecen ser métodos más confiables para evaluar el progreso terapéutico, en contraste con la entrevista de los padres que se basa en la estimación subjetiva de los tutores. Estos métodos serían significativamente más confiables si los examinadores fueran entrenados, calibrados y cegados. Además, se podría desarrollar un protocolo estandarizado para la entrevista con los padres. Por lo que, es muy importante desarrollar técnicas precisas, reproducibles y confiables para el examen y la evaluación de la función oral ${ }^{(17)}$.

Para el éxito del tratamiento es esencial que en cada etapa exista gran cooperación del paciente y principalmente de sus padres, ya que, el resultado final de la terapia siempre depende del compromiso de ellos. El TTFP es beneficioso, pero impone demandas adicionales a los niños y cuidadores ya sobrecargados, es por eso que el profesional debe motivarlos a que participen y supervisen cuidadosamente los efectos del tratamiento $^{(4)}$. En la literatura, los diferentes desarrollos interindividuales que se observan entre un niño con SD y otro, se atribuyen al cumplimiento variable por parte de los padres y pacientes al tratamiento ${ }^{(23)}$.

En general, se observa una heterogeneidad en la duración del tratamiento y la edad media de los participantes y el tiempo transcurrido posterior a la intervención. En los estudios incluidos en esta revisión, al inicio del tratamiento, la edad media de los participantes varió entre 2 meses y 12 ańos. Sin embargo, es bien sabido que la terapia de Castillo Morales enfatiza el tratamiento precoz para lograr la función motora oral normal en niños con $\mathrm{SD}^{(17)}$. Debido a que, el primer año de vida es el periodo de mayor desarrollo del sistema nervioso central y de la boca, es que el tratamiento debe ser iniciado lo más tempranamente posible ${ }^{(26)}$. Por otro lado, 
la duración del uso de la placa palatina fue entre 2 a 48 meses. Con las diferencias expuestas anteriormente, es difícil interpretar los hallazgos para la edad y duración del tratamiento ideal. Se sugiere que los estudios futuros realicen estandarización de la duración del tratamiento y edad de los nińos estudiados, para verificar el momento óptimo de intervención ${ }^{(17)}$.

\section{Conclusiones}

La evidencia sugiere que un niño con SD efectivamente se beneficia del TTFP, pues se observan mejoras de la función motora oral y expresión facial, aumento del cierre bucal y disminución de la protrusión lingual, observando mayores cambios en los casos más severos. Sin embargo, no se puede afirmar que con esta terapia exista un aumento real de la tonicidad muscular, pues para ello se requiere el uso de métodos objetivos que permitan cuantificarla.

Es necesaria la realización de nuevos estudios con muestras más representativas, métodos de evaluación estándar y objetivos, y con parámetros limitados en relación a la edad de inicio del tratamiento y la duración de éste.

\section{Contribución de autoría}

1. Concepción y diseño del estudio

2. Adquisición de datos

3. Análisis de datos

4. Discusión de los resultados

5. Redacción del manuscrito

6. Aprobación de la versión final del manuscrito

PV ha contribuido en: 1, 2, 3, 4 y 5 . FR ha contribuido en: 1, 2, 3, 4 y 5 .

GS ha contribuido en: 1, 2, 3, 4 y 5.

CF ha contribuido en: 1,5 y 6 .

MAP ha contribuido en: 5 y 6 .

\section{Referencias}

1. Desai S. Down syndrome: A review of the literature. Oral Surg Oral Med Oral Pathol Oral Radiol Endod.1997; 84 (3): 279-285.

2. Van Marrewijk DJ, Van Stiphout MA, Reuland-Bosma W, Bronkhorst EM, Ongkosuwito EM. The relationship between craniofacial development and hypodontia in patients with Down syndrome. Eur J Orthod. 2016; 38 (2): 178-83.

3. Nazer J, Cifuentes L. Estudio epidemiológico global del síndrome de Down. Rev Chil Pediatr. 2011; 82 (2): 105-112.

4. Matthews-Brzozowska T, Cudzilo D, Walasz J. Rehabilitation of the Orofacial Complex by Means of a Stimulating Plate in Children with Down Syndrome. Adv Clin Exp Med. 2015; 24 (2): 301-305.

5. Roizen N, Patterson D. Down's Syndrome: Seminar. Lancet. 2003; 361: 1281-89.

6. Marques LS, Alcântara CEP, Pereira LJ, Ramos-Jorge M. Down syndrome: a risk factor for malocclusion severity?. Braz Oral Res [online]. 2015; 29 (1): 1-7.

7. Shukla D, Bablani D, Chowdhry A, Thapar R, Gupta P, Mishra S. Dentofacial and Cranial Changes in Down Syndrome. Osong Public Health Res Perspect. 2014; 5 (6):339-344.

8. Morales M, Naukart Z. Prevalencia de maloclusiones en pacientes con Síndrome de Down. Oral. 2009; 10 (32): 537-539.

9. Bauer D, Evans CA, BeGole EA, Salzmann L. Severity of Occlusal Disharmonies in Down Syndrome. Int J Dent. 2012; 2012:1-6.

10. Suri S, Tompson B, Cornfoot L. Cranial base, maxillary and mandibular morphology in Down syndrome. Angle Orthod. 2010; 80 (5): 861-869.

11. Allareddy V, Ching N, Macklin EA, Voelz L, Weintraub G, Davidson E, Prock L, Rosen D, Brunn R, Skotko B. Craniofacial features as assessed by lateral cephalometric measurements in children with Down syndrome. Prog Orthod. 2016; 17 (1): 1-12.

12. Alió J, Lorenzo J, Iglesias M, Manso F, Ramírez E. Longitudinal maxillary growth in Down Syndrome patients. Angle Orthod. 2011; 81 (2): 252-259. 
13. López-Pérez R, Borges-Yáñez SA, López-Morales P. Anterior Open Bite and Speech Disorders in Children with Down Syndrome. Angle Orthod. 2008; 78 (2): 221-227.

14. Oliveira AC, Paiva SM, Campos MR, Czeresnia D. Factors associated with malocclusions in children and adolescents with Down syndrome. Am J Orthod Dentofacial Orthop. 2008; 133: 489.e1-489.e8.

15. Limbrock GJ, Fischer-Brandies H. Avalle C. Castillo Morales Orofacial Therapy: Treatment of 67 Children With Down Syndrome. Dev Med Child Neurol. 1991; 33: 296-303.

16. Chad L. Critical Review: What are the effects of palatal plate therapy on orofacial features and speech in children with Down syndrome?. [M.Cl.Sc SLP Candidate]. University of Western Ontario: School of Communication Sciences and Disorders. 2013.

17. Javed F, Akram Z, Barillas AP, Kellesarian SV, Ahmed HB, Khan J, Almas K. Outcome of orthodontic palatal plate therapy for orofacial dysfunction in children with Down syndrome: A systematic review. Orthod Craniofac Res. 2018; 21: 20-26.

18. Hohoff A, Ehmer U. Short-Term and LongTerm Results after Early Treatment with the Castillo Morales Stimulating Plate. J Orofac Orthop. 1999; 60 (1): 2-12.

19. Schuster G, Giese R. Retrospective Clinical Investigation of the Impact of Early Treatment of Children with Down's Syndrome According to Castillo-Morales. J Orofac Orthop. 2001; 62: 255-263.
20. Carlstedt K, Henningson G, Dallöf G. A fouryear longitudinal study of palatal plate therapy in children with Down Syndrome: effects on oral motor function, articulation and communication preferences. Acta Odontol Scand. 2003; 61: 39-46.

21. Carlstedt K, Henningson G, McAllister A, Dahllöf G. Long-term effects of palatal plate therapy on oral motor function in children with Down syndrome evaluated by video registration. Acta Odontol Scand. 2001; 59 (2): 63-68.

22. Carlstedt K, Dallöf G, Nilsson B, Modeer T. Effect of palatal plate therapy in children with Down syndrome A 1-year study. Acta Odontol Scand. 1996; 54 (2): 122-125.

23. Korbmacher H, Limbrock J, Kahl-Nieke B. Orofacial Development in Children with Down's Syndrome 12 Years after Early Intervention with a Stimulating Plate. J Orofac Orthop. 2004; 65: 60-73.

24. Bäckman B, Grevér-Sjölander AC, Bengtsson K, Persson J, Johansson I. Children with Down syndrome: oral development and morphology after use of palatal plates between 6 and 48 months of age. Int J Paediatr Dent. 2007; 17: 19-28.

25. Padró MJ, Barraza E, Brücher C, Concha E, Delgado V. Efectividad del Uso de Placas Palatinas y de la Estimulación Orofacial en el Desarrollo Oral en Nińos con Síndrome de Down. Rev Chil Pediatr. 2010; 81 (1): 46-52.

26. Carneiro V, Gonzales J, Fraiz F. Utilización de la placa palatina de memoria y desarrollo orofacial en infante con Síndrome de Down. Rev Cubana Estomatol. 2012; 49 (4): 305-311.

Paula Vivar: paula.vivar@gmail.com 